\title{
Staatliche Identitätsfindung und Sprache - Das Beispiel Somalia
}

\author{
von Thomas Labahn
}

Im Juli 1982 war in englischsprachigen Zeitungen von Demonstrationen im südindischen Bundesstaat Karnataka zu lesen (so die "Times" am 8. Juli 1982). Im Verlaufe dieser Unruhen eröffnete die Polizei das Feuer auf die Demonstranten; es gab Tote und Verwundete. Was waren die Ursachen dieser Zusammenstöße? Es ging nicht etwa um Lohnerhöhungen, niedrigere Lebensmittelpreise oder bessere Lebensbedingungen, sondern um eine angemessene Nutzung des Kannada, der dominanten Sprache in jener Region Indiens, als Unterrichtssprache in den Schulen.

Solche Gegebenheiten werden oft für entwicklungsländerspezifische Auswüchse gehalten. Dabei wird jedoch übersehen, daß es auch in Europa zu gewalttätigen Auseinandersetzungen hinsichtlich des Sprachgebrauchs zwischen verschiedenen Bevölkerungsgruppen bzw. zwischen Minoritätsgruppen und der zentralen Staatsgewalt gekommen ist. Man denke nur an Belgien, das Baskenland oder (in geringerem Maße) an die Bretagne. Eine Wurzel dieses Problems erfaßt Guggenberger sicherlich, wenn er über einen aufkeimenden Regionalismus in Europa schreibt: "Kennzeichen der Neuorientierung sind u. a. auch die Wiederentdeckung der Mundart und das "Bekenntnis" zur Region . . . Regionalismus und Dialekt sind Ausdrucksmittel einer weitverbreiteten radikalen Opposition, aber nicht nur dies: Sie vermitteln und aktualisieren auch einen verbindlichen Erfahrungshorizont und beschaffen den vom sozialen Wandel besonders hart Betroffenen die unentbehrliche Solidaritätsvergewisserung. " ${ }^{1}$

Der Widerstand gegen die Allmacht einer zentralen Staatsautorität läßt sich auf linguistischem Gebiet als Reflex auf historische Vorgänge interpretieren. Durch die Umwälzung des Produktionswesens sahen sich die Territorialstaaten Europas vom 16. Jahrhundert an veränderten Funktionszuschreibungen gegenüber, die in Zentralisierung und Bürokratisierung ihren Ausdruck fanden. Dabei blieb auch der Bereich der Sprache

Eine ausführliche Wiedergabe der sprachpolitischen Diskussionen in Somalia wie auch deren Einbettung in gesamtgesellschaftliche Zusammenhänge ist zu finden in: Labahn, Th., Sprache und Staat - Sprach politik in Somalia, Buske Verlag Hamburg 1982.

Einen umfassenden Uberblick über die somalische Geschichte bietet: Lewis, I. M., A Modern History of Somalia, Nation and State in the Horn of Africa, London 1980.

Auch wenn die Bewertung einzelner Fakten nicht immer derjenigen des Autors dieses Artikels entspricht, kann zur Vertiefung der verfassungsrechtlichen Situation in Somalia folgender Artikel empfohlen werden: Nuscheler, F., Politische Organisation und Repräsentation in Afrika - Somalia, in: Sternberger/Vogel/Nohlen/Landfried (eds.), Die Wahl der Parlamente und anderer Staatsorgane, Berlin/New York 1978, p. 1909-1943.

1 Guggenberger, B., Wertwandel und gesellschaftliche Fundamentalpolarisierung, in: Ellwein, Th. (ed.), Politikfeld-Analysen 1979, Opladen 1980, p. $271 / 272$. 
nicht ausgespart. Augenfälligstes Beispiel in diesem Kontext ist Frankreich. Dort nahm der Zentralismus direkten Einfluß auf den Sprachgebrauch, denn mit der Verordnung von Villers-Cotterêts des Jahres 1539 wurde Französisch zur offiziellen Sprache erklärt. Dies bewirkte einen schwindenden Gebrauch der einzelnen Regionalsprachen (z. B. Provencalisch und Bretonisch). Der politisch-administrative Zentralismus war Grundlage für den linguistischen Zentralismus.

Entsprechende Prozesse zu initiieren, ist seit geraumer Zeit das Bestreben der meisten Staaten Schwarzafrikas. Bezogen auf die Bevölkerungszahl gibt es in Afrika eine größere Zahl an Sprachen als in anderen Kontinenten, insgesamt sind es über 1000. Ist die linguistische Situation in den einzelnen Staaten auch heterogen, so sind die daraus erwachsenden Probleme doch ähnlich.

Wie Pool feststellte, existiert eine Korrelation zwischen der Anzahl der innerhalb der Landesgrenzen Verwendung findenden Sprachen und dem Entwicklungsstand des jeweiligen Staates: "A country that is linguistically highly heterogeneous is always underdeveloped and a country that is developed always has considerable language uniformity. $"{ }^{2}$ Welche Vorteile z. B. eine Nationalsprache mit sich bringt, sei durch einen Blick auf volkswirtschaftliche Implikationen verdeutlicht. Stellt die gesamte Bevölkerung eine linguistisch homogene Einheit dar, so können politische, ökonomische und soziale Prozesse besser und mit erhöhter Effizienz geplant werden. Eine kostenimplizierende Verdoppelung bzw. Multiplikation von Planung ist nicht notwendig. Sind demgegenüber mehrere Sprachgruppen in einem Staat vereint, so ist eine Vervielfachung der Verwaltungseinheiten die direkte Folge. Neben erhöhten Kosten bedeutet dies gleichzeitig eine Einschränkung der administrativen Flexibilität.

Damit ist bislang jedoch nur die instrumentelle Funktion von Sprache angedeutet worden. Durch ihren symbolischen Gehalt kommt ihr zusätzlich aber auch noch eine wesentliche Funktion hinsichtlich der Stabilität und der nationalstaatlichen Kohärenz eines Landes zu. So führt Kelman aus: "A national language serves as a major object and symbol of attachment by bridging immediate loyalities with transcendent ones. It is through his mother tongue that the child is exposed to the songs, the heroic tales, and the traditional wisdom of his people. The very language in which he communicates with his mother and with his immediate environment also serves to link him with a wider group, most of whose members are distant in time and place. Language thus provides a continuity and scope without which a sense of overarching nationality could not be constructed. ${ }^{3}$ Vor dem Hintergrund dieser Ausführungen wird offenbar, welch schwere Last die sprachliche Heterogenität den jungen Staaten Afrikas nach Erlangung der Unabhängigkeit aufbürdete. Als es darum ging, die Entscheidung über das offizielle Medium im Lande zu treffen, konnte man sich auf keine der Landessprachen einigen. Logische Folge

2 Pool, J., National Development and Language Diversity, in: Fishman, J. A. (ed.), Advances in the Sociology of Language, Den Haag 1971, p. 213.

3 Kelman, H. C., Language as a n Aid and Barrier to Involvement in the National System, in: Rubin/Jernudd (eds.), Can Language be Planned?, Honolulu 1971, p. 31. 
war, daß die Sprache der ehemaligen Kolonialmacht offiziellen Status erhielt und in Schule und Verwaltung, vor Gericht und in den Massenmedien Verwendung fand. Dies lag auch im Interesse der herrschenden Eliten, die über entsprechende Fremdsprachenkenntnisse verfügten und somit ihren Einflußbereich wirksam abschirmen konnten; breiten Kreisen der Bevölkerung hingegen wurde die Einflußnahme auf öffentliche Anliegen nicht nur erschwert, sondern unmöglich gemacht. Die momentan zu beobachtende Klassenstruktur in den afrikanischen Staaten ist in nicht unbedeutendem Maße Auswuchs unterschiedlicher sprachlicher Fremdsprachenkompetenz in der Bevölkerung, und sie wird auch weiterhin durch die klassenspezifisch bedingten Möglichkeiten des Spracherwerbs perpetuiert. Die sprachliche Zerrissenheit stellte einen der wesentlichen Gründe dar, daß die vielbeschworene staatliche Identitätsfindung für afrikanische Staaten eine Fiktion blieb.

Somalia zählt zu den wenigen afrikanischen Staaten, in denen eine Landessprache als offizielles Medium benutzt wird. In Somalia geschieht dies seit nunmehr zehn Jahren. Zuvor stellte sich die Situation auch dort ähnlich den zuvor skizzierten Zusammenhängen dar.

Als 1960 nach dem Zusammenschluß des englischen und des italienischen Kolonialgebietes die Republik Somalia entstand, entschied man sich dafür, den Sprachen der ehemaligen Kolonialmächte sowie dem Arabisch den Status einer offiziellen Sprache zu geben, und dies obwohl die Landessprache Somali eine 97 \%ige Verbreitung unter der Bevölkerung besitzt. Ursächlich verantwortlich für diesen paradox erscheinenden Beschluß war die Tatsache, daß sowohl innerhalb der politischen Führung wie auch in der Bevölkerung großer Dissens bestand, welche der zur Disposition stehenden Schriften für das Somali, das über keinerlei standardisierte Schriftform verfügte, adaptiert werden sollte. Ohne Schrift bestand jedoch keine Möglichkeit, das Somali zur offiziellen Sprache zu machen. In der Folgezeit wurde die Entscheidung um die Schrift immer wieder verzögert. Wie und warum 1972 die Umsetzung einer staatlich konzipierten Sprachpolitik gelang, soll im Folgenden nachgezeichnet werden. Interessanterweise läßt sich dabei eine Kongruenz in der Entwicklung von sprachpolitischen Intentionen und gesellschaftlichem System sowie der Herausbildung staatlicher Strukturen belegen. Unter Anlegung eines groben Rasters sind vier Phasen zu unterscheiden: eine erste, archaische schloß mit Beendigung des 2. Weltkrieges ab; die zweite ist charakterisiert durch eine sich abzeichnende Neuorientierung und eine Aktualisierung des Staatsgedankens und geht 1960 mit Erlangung der Unabhängigkeit nahtlos in einen dritten Abschnitt über, der in den ersten Jahren entscheidend zur Formung des somalischen Nationalgedankens beiträgt. Mit der Machtübernahme der Militärs beginnt 1969 die vierte Phase, in der es zur Instrumentalisierung des Nationalgefühls der Somalis und zu einer Ausweitung des staatlichen Strukturnetzes kommt.

Mit einer Einwohnerzahl von ca. 4 Millionen stellt Somalia den nordöstlichen Zipfel Afrikas dar. Die ethnisch-kulturelle Homogenität der Bevölkerung ist eines der augenfälligsten Kennzeichen dieses nach UN-Statistiken zu den ärmsten Staaten der Welt gezählten Landes. - Beziehungen zur Außenwelt bestehen seit altersher, insbesondere der 
Einfluß der arabischen Halbinsel spielte eine bedeutsame Rolle. So konnte sich der Islam frühzeitig ausbreiten und die autochthonen Naturreligionen schon bald gänzlich überlagern. Die Geschichte der letzten eintausend Jahre verlief für die Somalis wechselvoll. Erst lösten sich Araber, Türken und Portugiesen als Machthaber ab; ihre Herrschaft beschränkte sich jedoch auf den Küstenstreifen und die wenigen Städte im Inland. Eine Penetration des Hinterlandes gelang keiner ausländischen Macht. Die Halbwüsten und Dornensavannen, in denen bis in die Neuzeit der überwiegende Teil der Somalis lebte, boten auch nur wenig Anreiz und so blieben die auf Nomadismus aufbauenden Lebensverhältnisse über Jahrhunderte weitgehend unbeeinflußt.

In eine Vielzahl von Clans und Subclans zersplittert, zogen die Somalis in akephalen Familienverbänden durch das Land, stets auf der Suche nach günstigen Weideplätzen für ihr Vieh. Selbst als am Ende des letzten Jahrhunderts Franzosen, Engländer und Italiener ihre Kolonien auf somalischem Siedlungsgebiet etablierten, volizogen sich nicht jene Änderungen im soziokulturellen System wie sie aus anderen Kolonialterritorien belegt sind. Auch das System der Nachrichtenübermittlung blieb während dieser Zeit unverändert. Informationen wurden mündlich weitergegeben, schriftliche Fixierungen nur in seltenen Fällen vorgenommen, denn Schreib- und Lesekenntnisse beschränkten sich auf eine dünne Schicht der Bevölkerung (Geistlichkeit und Kaufleute). In solchen Fällen bediente man sich der arabischen Schrift, die durch den Gebrauch in den Koranschulen eine gewisse Verbreitung erfuhr. Da es durch die phonetischen Gegebenheiten des Somali jedoch Schwierigkeiten für eine vollständige Ưbertragbarkeit gab, erfuhr diese Schriftform nie eine Standardisierung. Dies ist auch nicht verwunderlich, da sich die Notwendigkeit zum Gebrauch von Schrift auf einen engen Kontext beschränkte, denn es ist von der Tatsache auszugehen, daß die Existenz einer genormten Schriftform an spezifische gesellschaftliche Grundstrukturierungen gebunden ist. So bestätigen denn auch alle bisher bekannten Schriftsysteme die Vermutung, daß eine positive Korrelation zwischen Seßhaftigkeit, der Herausbildung politischer Strukturen und einer arbeitsteiligen Produktionsform einerseits und der Entwicklung eines Schriftsystems andererseits besteht. Schmitt bemerkt dazu: "Die Anlässe, Mitteilungen oder Notizen aufzuzeichnen, mehren sich, wenn die Gruppe eng beieinander lebender Menschen sich vergrößert und schließlich Städte entstehen mit vielseitig ausgeprägten Wirtschafts- und Rechtsverhältnissen." ${ }^{4} \mathrm{Da}$ keine dieser Bedingungen in Somalia gegeben war, bestand auch nie die Notwendigkeit der Einführung eines elaborierten Schriftsystems. Zusätzlich muß erwähnt werden, daß kompensatorisch das System mündlicher Kommunikation so hoch entwikkelt wurde, daß es den gesellschaftlichen Bedürfnissen an Informationsvermittlung entsprach. Auf seiner Grundlage entwickelte sich eine meritokratische Kaste von Barden und Nachrichtenträgern. Der Stolz, der auch heute noch über die oralen Traditionen in Somalia zu finden ist, stellt ein Relikt jener Zeit ohne Schrift für die Muttersprache dar. 
Wenngleich die Präsenz der Kolonialmächte auch nicht übermäßig spürbar war und sich auf die Städte beschränkte, so gab sie doch mancherlei Impulse. In diesem Zusammenhang spielte das Erziehungswesen eine bedeutsame Rolle. Zwar gab es seitens der Italiener und Briten - entsprechend der nur rudimentär ausgebildeten Verwaltungsstrukturen - nur geringe Bestrebungen in diesem Bereich, doch trugen diese entscheidend dazu bei, das Problem der Schriftlosigkeit ihrer Muttersprache im Bewußtsein, zumindest bei einem Teil, der Somalis zu verankern. Und so ist es nicht verwunderlich, wenn speziell aus den ersten Jahrzehnten dieses Jahrhunderts verschiedene Versuche belegt sind, entweder Standardisierungen der arabischen Schrift für das Somali vorzunehmen oder vollständig neue Schriftsysteme zu entwickeln. Der Verbreitungsgrad dieser Innovationen blieb jedoch meist regional begrenzt. Die weiteste Beachtung erlangte die nach ihrem Erfinder benannte Osmania. Doch auch sie fand keine landesweite Verbreitung.

Die Bemühungen der Europäer, das lateinische Schriftsystem einzuführen, scheiterten am Unwillen der Bevölkerung. Zutiefst dem islamischen Glauben verbunden, sah man in den Vorhaben der Kolonialadministration den Versuch, den Einfluß des Islam zu schwächen. Wie stark dieses Thema emotional aufgeladen war, beweist die Tatsache, daß der erste Director of Education, als er 1936 die Fortschritte am Bau einer von den Briten finanzierten Schule in Burao besichtigen wollte, mit einem Steinhagel durch die Bevölkerung begrüßt wurde. Daraufhin wurde das Projekt gestoppt. Als ein Jahr später der erneute Versuch einer Schuleröffnung unternommen wurde, kam es zu Demonstrationen und Unruhen, in deren Verlauf mehrere Somalis getötet wurden:- Abschließend läßt sich feststellen, daß die Strukturen in Somalia sich bis zum 2. Weltkrieg gegenüber den vorherigen Jahrhunderten nur wenig verändert hatten.

Nach Kriegsende begann eine neue Phase, die eine langsame, doch stetige Änderung der gesellschaftlichen Strukturen mit sich brachte. Es kam zur Gründung des Somali Youth Club, der den ersten politischen Zusammenschluß im Lande darstellte und sich 1947 in Somali Youth League (SYL) umbenannte. Seine Mitgliedschaft rekrutierte der Club insbesondere aus einer zahlenmäßig immer stärker werdenden Gruppe von westlich beeinflußten, in der Verwaltung und der Polizeitruppe tätigen Somalis. Die Gründung kennzeichnet einen wesentlichen Umschwung. Propagiert wurde nunmehr der Gedanke eines der Moderne zugewandten unabhängigen Staates Somalia. Ziel war es dabei, die traditionelle Clangebundenheit des Individuums zu überwinden und einen alle Somalis vereinenden Nationalismus an seine Stelle treten zu lassen. Verbesserte Ausbildungsmöglichkeiten im allgemeinen sowie die Einführung einer Schrift waren zwei Programmpunkte, in denen sich diese Zielsetzungen widerspiegelten. Daß man sich für das Schriftsystem Osmania aussprach, kann nicht verwundern, konnte dieses doch am Besten als nationales Symbol herausgestellt werden.

War in den Jahren zuvor das Desinteresse für und die Indifferenz gegenüber den bestehenden Schriftformen in weiten Teilen der Bevölkerung groß gewesen, so führte das nun aufblühende nationale Bewußtsein zu einer Wende. Die Befürwortung einer wahrhaft eigenständigen somalischen Schrift entsprach dem damaligen von der SYL getragenen Patriotismus. 
Doch ein Wechsel in der Spitze der SYL führte ab 1949 zu einer veränderten Situation: die einen somalischen Nationalismus propagierende Gruppe wurde durch Befürworter pan-arabischer bzw. pan-islamischer Vorstellungen abgelöst. Dadurch verlor die Osmania, bisher Ausdruck des Nationalstolzes und als ein Mittel im Prozeß der Dekolonisation verstanden, an Bedeutung. Nach dem Willen der pro-arabischen Kreise um den SYL-Präsidenten Haji Mohamed Hussein sollte die arabische Sprache zum offiziellen Medium in Somalia erhoben werden, das landesweit verbreitete Somali nur noch die Rolle einer Vernakulärsprache spielen. Damit, so die Argumentation, sei automatisch auch das Problem der Schrift gelöst. Zudem sei durch die Kenntnis des Korans schon eine umfassende Kenntnis des Arabischen in der Bevölkerung gegeben. Diese Forderung fand die volle Unterstützung der islamischen Geistlichkeit; für viele Somalis hingegen war ein solcher Rückgriff auf das Arabische gleichbedeutend mit einer Aufgabe ihres Identifikationsgefühls und des gerade erwachten Nationalbewußtseins. Damit standen sich zu einer Zeit als erstmals Rundfunk und Printmedien Einfluß auf die öffentliche Meinungsbildung nahmen, zwei Blöcke gegenüber, die beide den Maßstab ihres Handelns aus wertrationalen Kriterien zogen: Nationalisten und islamische Fundamentalisten. Eingedenk ihrer negativen Erfahrungen in den Jahrzehnten zuvor vermieden beide Kolonialmächte jegliche Stellungnahme zu diesem Thema und erklärten, daß ein Entscheid über die einzuführende offizielle Sprache und Schrift den Somalis nach Erlangung der Unabhängigkeit obläge.

So blieb diese Diskussion bis 1960 in unverminderter Schärfe virulent. In der SYL kam es zu erbitterten internen Auseinandersetzungen, doch diese waren eher ein Abbild einer sich am jeweiligen Claninteresse ausrichtenden Politik als das Bemühen, mittels zweckrationaler Strategien eine Problemlösung anzustreben. Der Versuch, mit tradierten Mitteln den Erfordernissen der Neuzeit zu begegnen, mußte scheitern. Dies erwies sich hinsichtlich der Sprach- und Schriftfrage als besonders folgenschwer, denn es blockierte nicht nur eine Entscheidung noch während der Kolonialzeit, sondern gab auch die Rahmenbedingungen für die Behandlung dieser Frage nach 1960 vor.

Zum gleichen Zeitpunkt setzte überdies eine Entwicklung ein, die die Situation noch mehr verwirrte. Die schrittweise Somalisierung des Verwaltungsapparates, d. h. die Besetzung relevanter Stellen mit Einheimischen, trug zur Bildung einer Gruppe bei, die sich offen zu einer Einführung der lateinischen Schrift bekannte. Obwohl sie anfangs zahlenmäßig nur eine geringe Stärke aufwies, blieb ihre Stimme nicht ungehört, da ihre Mitglieder einflußreiche Positionen bekleideten.

Mit dem Slogan "Laatin waa laa Diin" (Latein ist ohne Gott) ging die islamische Geistlichkeit gegen diese Bestrebungen vor und war damit auch erfolgreich. Denn als Abdilahi Isse, erster Premierminister einer sich ausschließlich aus Somalis zusammensetzenden Regierung im italienischen Treuhandgebiet, 1957 ohne vorherige Absprache mit der Führungsspitze seiner Partei eine Seite mit Berichten in Somali, geschrieben in lateinischer Schrift, in den "Corriere della Somalia" einrücken ließ, schlugen ihm vehemente inner- wie außerparteiliche Widerstände entgegen. Eine Fortführung des Projektes war unmöglich. 
So läßt sich feststellen, daß sich während dieser zweiten Phase die individuellen, vereinzelten Versuche hinsichtlich einer Schrifteinführung in den vorherigen Jahrzehnten verbunden mit der Frage nach der offiziellen Sprache des Landes zu einer Komplexität entwickelt hatten, die mit den gängigen Konfliktbewältigungsmustern nicht gelöst werden konnte. Zwar war man sich während dieses Zeitraums, der die Vorstrukturierung der staatlichen Einheit zu bewältigen hatte, bewußt, daß die Zukunftsprobleme nur durch die Erhöhung der Informationsaufnahme- und verarbeitungskapazität bewältigt werden könnten, doch nach einer ersten Phase der Kohärenz, in der der latente Nationalismus für politische Zielsetzungen genutzt wurde, verhinderte der Rückfall in traditionelle Bezugsmuster das Beschreiten eines zukunftsträchtigen Weges.

Die Behandlung der Frage in der maßgeblichen Partei war blockiert, der Druck und das Interesse seitens der Bevölkerung zu schwach, als daß durch sie eine Lösung hätte herbeigeführt werden können.

Die somalischen Hoffnungen beruhten jedoch auf der Zeit nach Erlangung der Unabhängigkeit. Wirtschaftswachstum, Modernisierung aller Lebensbereiche sollten die Entwicklung des Landes bestimmen. Und es war weitverbreitete Ansicht, daß es in diesem Rahmen auch zu einer Klärung der Frage um die offizielle Sprache und die einzuführende Schrift kommen würde.

Die nationale Unabhängigkeit brachte nicht den erhofften sozialen Fortschritt; zu gering waren die Ressourcen, um die infrastrukturelle Erschließung, den Aufbau der heimischen Wirtschaft und letztlich einen die gesamte Gesellschaft erfassenden Transformationsprozeß vorantreiben zu können. Doch es waren nicht allein die fehlenden materiellen Voraussetzungen, die die notwendige Entwicklung behinderten. Gleichermaßen entscheidend war der Unwille der somalischen Politiker, mit überkommenen Strukturen zu brechen. Der zwischen allen Parteien bestehende politische Fundamentalkonsens wurde durch eine auf der Clanzugehörigkeit basierenden Ausgewogenheit bei der Besetzung von Ämtern erzielt. Strittige Fragen blieben ausgeklammert.

Als Beispiel für die Konzeptionslosigkeit der nächsten Jahre bei der Behandlung relevanter politischer Anliegen kann die Sprach- und Schriftproblematik dienen. War es auf der einen Seite der latente Nationalismus der die Staatsgründung erleichterte und offene Verteilungskämpfe zwischen verschiedenen Bevölkerungsteilen unterband, so war auf der anderen Seite ein Erstarken des Nationalismus als direkter Reflex auf diesen formalen Akt der Staatsgründung sichtbar. Das wirkte sich auch auf die Sprachfrage aus. Vor dem Hintergrund einer alle Bevölkerungsteile umfassenden nationalen Begeisterung hatten die pan-arabischen Kreise jenen Spielraum verloren, der ihnen zuvor die Propagierung einer Einführung der arabischen Sprache erlaubt hatte. Daher gaben sie diese Bemühungen umgehend auf und beschränkten sich darauf, für eine Adaption der arabischen Schrift für das Somali zu plädieren. Damit hatte sich die Anzahl der im Widerstreit befindlichen Positionen verringert, war eine Reduzierung auf die Schriftproblematik erfolgt, doch da die Voraussetzungen für den Gebrauch der Landessprache Somali als offiziellem Medium aufgrund der noch fehlenden Verschriftung immer noch nicht gegeben waren, entschied sich die politische Führung für die Sprache der ehemaligen Kolo- 
nialmächte und das Arabische. Das Nebeneinander dreier offizieller Sprachen und die Tatsache, daß für die Aufnahme in den Verwaltungsdienst lediglich die Beherrschung einer der offiziellen Sprachen unabdingbare Voraussetzung war, schufen in der Administration schwerwiegende Probleme. Konnten Verhandlungen in den Ministerien auch in Somali geführt werden, so ergaben sich notgedrungenerweise Søhwierigkeiten, wenn die schriftliche Abfassung von Dokumenten anstand und die mit der Sache befaßten Beamten unterschiedliche Fremdsprachen beherrschten. In solchen Fällen mußten stets Dolmetscher eingeschaltet werden, was für den Staatshaushalt eine erhebliche finanzielle Belastung darstellte.

So wurde von der ersten unabhängigen somalischen Regierung auch betont, daß dieser Zustand nur als Úbergangslösung zu verstehen sei und von ihr angestrebt werde, dem Somali baldmöglichst offiziellen Status zu verleihen. Entsprechende Passagen finden sich in der Regierungserklärung von Premierminister Abdirashid Shermake. Als positives Zeichen wurde es dann auch allseitig gewertet, daß die Regierung am 18. Oktober 1960 die Einsetzung einer Sprachkommission beschloß und diese noch im gleichen Monat durch Erziehungsminister Ali Garad Jama einberufen wurde. Da in ihr jedoch Exponenten der verschiedenen zur Disposition stehenden Schriftsysteme vertreten waren, war ein Scheitern vorgezeichnet. Zwar wurde sogleich eine zweite Kommission einberufen, doch als diese in ihrem Abschlußbericht der lateinischen Schrift den Vorzug gab, stand sie mit dieser Auffassung konträr zu den Vorstellungen der damaligen Regierungsspitze, die die arabische Schrift favorisierte. Der Report verschwand ohne parlamentarische Erörterung in den Ablagen des zuständigen Ministeriums.

Zwar war es auch in den folgenden Jahren stets erklärtes Ziel aller Regierungen dem schriftlosen Zustand der Muttersprache ein Ende zu bereiten, doch einen ernsthaften Versuch unternahm nur noch der zukunftsorientierte Premierminister Abdirazak Haji Hussein, der sich offen für die lateinische Schrift aussprach. In einem Gespräch erklärte er: "I can see how easy it is for those who do not have to take lecture notes, draft memos, type letters, write reports and so on - at least not every day in their lives. I can see how easy it is for them to advocate Osmania or Arabic for chauvinistic or pseudo-religious reasons. But we have to be pragmatic. We have to look from the angle of the man who is forced to use the written language almost daily in his life. To such a man, a script is not like an ornament, like the Somali rugs we hang in our houses for decoration . . . ${ }^{5}$ Doch bevor er sich mit diesem Pragmatismus durchsetzen konnte, war er Anfang 1967 in seinem Amte abgelöst worden.

Blieben die Strukturen auch unverändert, gab es weiterhin Befürworter für die Einführung der arabischen, der lateinischen und einer somalischen Schrift, so vollzogen sich doch Verschiebungen in der Gewichtung der einzelnen Gruppen zueinander. Die Anhänger eines somalischen Schriftsystems verloren an Bedeutung, während die Gruppe, die sich für die lateinische Schrift aussprach, zusehends an Einfluß gewann. Daß die natio-

5 Hussein M. Adam, A Nation in Search of a Script: The Problem of Establishing a National Orthography for Somali, M. A. thesis, Makerere University of East Africa 1968, p. 146. 
nalistisch gesonnenen Kräfte an Boden verloren, ist in Zusammenhang mit der Unfähigkeit des Staates zu sehen, eine Verbesserung der Lebensverhältnisse für seine Bürger herbeizuführen, was vor dem Hintergrund eines korrupten, sich an Gruppenpartikularismen ausrichtenden Parlaments auch keine Verwunderung erzielt.

Um als Gesamtsystem effektiv zu funktionieren, ist es für den Staat wesentlich, daß die von ihm verfolgten Zielsetzungen und damit einhergehende spezifische Ansprüche und Forderungen des politischen Systems als legitim anerkannt und von der Bevölkerung akzeptiert werden. Da der Staat mit seinen Institutionen in Somalia unfähig blieb, die physische und soziale Sicherheit für alle Bürger zu garantieren, waren die alten Clanstrukturen jenes Systems, dem sich der Einzelne im höheren Maße verpflichtet fühlte als dem Staat. Anfänglich versuchte man mangelnde innenpolitische Erfolge durch die gegenüber dem Nachbarn Äthiopien vorgebrachten, historisch gerechtfertigten Ansprüche auf den Ogaden kompensatorisch auszugleichen. Vorübergehend schufen sie auch Mobilisierung und trugen zur Identifikation mit dem politischen System auf breiter Basis bei. Das Scheitern dieser Politik der Stärke führte jedoch zu einem weiteren Absinken der integrativen Affinität gegenüber dem Staatswesen. Bis zu einem gewissen Grad kann eine gegenseitige Substituierung von affektueller und instrumenteller Affinität erfolgen. In Somalia hatte der Staat jedoch in beiden Bereichen versagt. Mangelnde Loyalität und ein Rückbezug auf die traditionellen Sozialstrukturen waren die Antwort der Bevölkerung. Blieb die Entwicklung des Staatswesens im Ganzen hinter den Notwendigkeiten zurück, so ist es nicht erstaunlich, daß es auch in der Schriftf rage zu keinerlei Entscheidung kam. Keine Regierung war willens ihre Machtbasis durch eine intensive Behandlung dieses emotionsgeladenen Themas zu erschüttern. War zuvor die Erwähnung noch Pflichtübung gewesen, so wurde 1967 dieser Komplex erstmals aus einer Regierungserklärung verbannt. Umfragen aus jenem Jahr belegen, daß die Hoffnung auf eine baldige Klärung über die Frage der Schrift in der Bevölkerung geschwunden war.

Als Präsident Shermake im Oktober 1969 einem Attentat zum Opfer fiel und die Armee vor der Benennung eines Nachfolgers am 21. Oktober nach einem unblutigen Staatsstreich die Macht übernahm, begründete das sich unter Führung von Generalmajor Siyad Barre konstituierende Supreme Revolutionary Council (SRC) seinen Schritt mit dem Vorwurf des Tribalismus, der Korruption, der Ungerechtigkeit und der Mißwirtschaft und traf damit auf Zustimmung bei der Mehrheit der Bevölkerung.

Erklärtes Ziel der zukünftigen Politik war die Transformation des gesamten gesellschaftlichen Systems. Dabei wird der Schriftfrage eine wesentliche Rolle zugestanden, denn nur auf der Grundlage eines geschriebenen Somali ist die Möglichkeit von Alphabetisierungskampagnen für die breite Masse der Bevölkerung möglich. So finden sich dann auch in der revolutionären Charta der neuen Machthaber folgende Passagen: Es sei vordringlich "to liquidate illiteracy and to develop an enlightened patrimonial and cultural heritage of the Somali people" und "to constitute, with appropriate measures the basic development of the writing of the Somali language."

Wer jetzt allerdings einen sofortigen Entscheid in dieser Frage erwartet hatte, wurde enttäuscht. Im ersten Jahr der Regierungstätigkeit der Militärs blieb es bei unverbindlichen 
Bemerkungen, die sich durch nichts von den Verlautbarungen der parlamentarischen Regierungen im Jahrzehnt zuvor unterschieden. Zwei Tatbestände waren dafür verantwortlich: Zum einen wurde anderen Bereichen Priorität für die Bewältigung der Zukunftsprobleme eingeräumt, zum anderen war das Meinungsbild innerhalb der politischen Führungsgremien nach der Machtübernahme ebenso gespalten wie in der Gesamtbevölkerung: es gab Befürworter der arabischen und der lateinischen Schrift wie der Osmania. Tiefgreifende Zerwürfnisse wurden vermieden, indem die Entscheidung über diese Frage verschoben wurde. Stattdessen wurde eine Kommission einberufen, der allerdings nicht die Aufgabe übertragen wurde, über die Vor- und Nachteile der einzelnen Schriftsysteme zu diskutieren, sondern Materialien für die Erstellung von Lehrbüchern für den Schulunterricht und die Erwachsenenbildung zu sammeln. Die Entscheidung über die letztlich einzuführende Schrift behielt sich der SRC vor.

Zum weiteren Verständnis des Ablaufes ist es notwendig, das Schriftproblem nicht als isolierte Einheit zu betrachten; es steht vielmehr in einer engen zeitlichen wie inhaltlichen Abfolge mit anderen Staats-Kampagnen und dem vom SRC propagierten Neuaufbau des Staates. Betont wurden soziale Gleichheit und Gerechtigkeit; die Schwerpunkte auf ökonomischem Gebiet sollten auf zentraler Planung und einer Ausweitung des Staatssektors liegen. Eine grundlegende Änderung der Verhältnisse war jedoch nur durch die Schaffung eines funktionstüchtigen Staatsapparates möglich. Die Realisierung dieses Zieles konnte nur durch die Stärkung der Staatsgewalt erfolgen, wobei auch das physische Machtmonopol eingesetzt wurde. Inhaltlich versuchte man, das Schlagwort des gesellschaftlichen Wandels durch Mobilisierung und Partizipation der Bevölkerung zu erreichen. Im ganzen Land wurden "Orientations Centres" gebaut. Sie sollten die Möglichkeit einer direkten Kommunikation zwischen der Bevölkerung und der politischen Führung bieten, darüberhinaus aber auch der Ort sein, an dem eine politische und ideologische Schulung vorgenommen werden konnte.

Schrittweise wurden einzelne Programmpunkte in Angriff genommen, wobei es zu einer Neubewertung möglicher Handlungsalternativen kam. Das Nationalbewußtsein der Somalis wurde erstmals in umfassendem Maße als materielle Ressource eingesetzt. So stellte auf ökonomischem Gebiet die Verfolgung eines autozentrierten Entwicklungsweges auf das ganzheitliche Bewußtsein der Bevölkerung um ihre ethnische Homogenität ab. Um jedoch die gewünschten Erfolge erzielen zu können, mußten jene gesellschaftlichen Strukturen eliminiert werden, die dieser Zielvorgabe diametral entgegenstanden. So ist es auch logisch, daß sich die ersten Staats-Kampagnen gegen Clan-Denken, Nepotismus und Korruption wendeten.

War bis 1969 das Auffangen des Konfliktpotentials für das politische System durch Patronage geglückt, so war es jetzt nicht länger die clanbezogene Ein- oder Anbindung, die die Unterstützung und die Loyalität der Bevölkerung sichern sollte, sondern ein leistungsbezogener Katalog verschiedener Kriterien. Dies war eine riskante Strategie, denn im Gegensatz zu den Zivilregierungen, die sich ihre Uberlebenschance durch die Respektierung der traditionellen Sozialstruktur gesichert hatten, sah sich das Militärregime einem von ihm selbst geschaffenen Erfolgsdruck unterworfen. Zwar konnte man auf die 
anfängliche Unterstützung durch die Bevölkerung bauen, doch die Gewinnung von Loyalitäten bei gleichzeitiger Abschaffung der alten Bezugsmuster gelang letztlich dadurch, daß die neuen staatlichen Strukturen sich in starkem Maße als Träger der individuellen Interessen des Einzelnen unter dem Vorzeichen einer allen Bevölkerungsteilen dienenden staatlichen Entwicklung darstellten und als solche auch akzeptiert wurden. So bot sich der politischen Führung 1972 die Möglichkeit, endgültig eine Schrifteinführung ins Auge zu fassen. Eine langfristige psychologische Einwirkung auf die Bevölkerung hatte zusätzlich die notwendigen Voraussetzungen geschaffen. Die Schriftfrage wurde in die Reihe anderer nationaler Anliegen wie den Aufbau eines sozialistischen Staatswesens, den Ausbau des landwirtschaftlichen Sektors, die Verbesserung im Schulwesen etc. eingereiht. Gleichzeitig war man sich seitens des SRC und der Regierung aber auch bewußt, daß das Konfliktniveau dieses explosiven Komplexes abgesenkt und die Konsensbildungsfähigkeit gesteigert werden mußte. Daher entzog man die Diskussion um die Schrift dem öffentlichen Forum. Barre verwies zwar zuweilen in Reden auf dieses Thema, verkündete Fortschritte in seiner Behandlung, doch eine Entfachung der Diskussion in der Bevölkerung wurde vermieden. Gleichwohl war man sich im SRC des Entscheidungsdrucks und des "Legitimationszwanges" (Elsenhans) bewußt. Als dann im engsten Führungszirkel um Barre die Entscheidung zugunsten der lateinischen Schrift fiel, wurde SRC-interne Opposition durch den Verweis auf die Notwendigkeit einer Kontinuität von revolutionären Kampagnen erstickt. Die Ausschaltung der zu erwartenden Opposition durch die islamische Geistlichkeit gelang durch die Androhung, daß für jenen Fall eine Konfizierung ihres Besitzes erfolgen würde.

Damit waren die Voraussetzungen gegeben, die Entscheidung zu verkünden. Am dritten Jahrestag der Revolution, am 21. Oktober 1972 war es soweit. Vor den versammelten Massen verkündete Barre die Entscheidung zugunsten der lateinischen Schrift, betonte jedoch gleichzeitig, daß der Gesichtspunkt einer sofortigen Einführung des Somali als offizieller Sprache im Vordergrund dieses Beschlusses gestanden habe und daß technisch-instrumentelle Gesichtspunkte den Ausschlag gegeben hätten: "I also want to impress on the Somali people today that an unanimous decision has been reached: to write the Somali script, to write in our mother tongue from today and to use it in matters of administration. A modified Latin script has been chosen for economic reasons as well as convenience. The resources of this nation cannot shoulder the burden of innovating a new alphabet (for matters of printing etc.) and there is also the inconvenience of having to wait for a long time before we realize this goal. If I may tell you, what this involves: it will make our educational system uniform. All the world is interrelated or linked by certain things like trade, sharing of knowledge and know-how: no country can afford to isolate itself. Most of the world uses the Latin alphabet. Every society wants its language to be developed and be learnt by many people. If we use a totally new script it would have become an isolated one. At the same time it has a particular value or importance in that it was seen as an easy alphabet which can been taught and spread quickly." Durch diese Schwerpunktverlagerung gelang es, die Entscheidung als revolutionär-nationales Anliegen darzustellen, zumal sie nur als Durchgangsstation zu verstehen sei, die die Kontinui- 
tät des Staatsaufbaus sichern würde. Die damit angesprochenen Alphabetisierungskampagnen konnten aufgrund der Tatsache, daß die notwendigen Materialien durch die Arbeit der Sprachkommission schon zur Verfügung standen, dann auch ab 1973 durchgeführt werden.

Hält man sich den Verlauf der sprachpolitischen Diskussion in Somalia vor Augen, so lassen sich folgende Feststellungen treffen: Sprache besitzt zweifellos einen herausgehobenen Stellenwert im Leben einer staatlichen Gemeinschaft, doch in ihrer politischen Behandlung scheint sie sich nicht grundsätzlich von anderen gesellschaftlichen Konfliktfeldern zu unterscheiden. Daß allein das Vertrauen, die magischen Kräfte einer gemeinsamen Sprache würden automatisch die Grundlagen für ein funktionierendes Staatswesen schaffen, nicht ausreichend ist, wurde durch die Vorgänge in Somalia nach 1960 evident. Eine sinnvolle Nutzung dieses Potentials erfolgte erst nach 1969, als unter Opferung wertrationaler Bezüge bzw. durch deren funktioneller Nutzung, Entscheidungen vorangetrieben wurden, die zuvor durch die gesellschaftlichen Strukturen verhindert worden waren. 
mand for notary services. The re-establishment of a network of public notary offices is considered part of strengthening the "Socialist Legal System". Public notary offices are state offices, supervised by the departments of justice of the People's Governments at all levels and are responsible for the official authentication and verification of documents and contracts and certain other legal matters. By June 1982, around 1,600 public notary offices had been set up in Chinese counties, districts and cities. This number now exceeds the highest number of such offices existing before the 'Cultural Revolution', around 1,100 in 1957 . So far, the public notary offices have mainly handled international cases, i.e. documents concerning the affairs of non-residents (mostly overseas Chinese) or nonChinese residents. But domestic cases are becoming more and more important.

The article presents a short introduction to the development of the system of public notary offices since 1949, and it further discusses the organization, functions and duties of these of fices, as well as regulations concerning the form of notarial documents. Some statistical data relating to the development between 1979 and 1982 are also given. A German translation of the "Provisional Regulations" is appended.

\section{The Search for National Identity and Language Problems - Somalia's Experience}

\section{by Thomas Labahn}

There are well over 1000 languages in Africa today. Thus African states are faced with the necessity to create official languages which, among other things, promote a sense of overarching nationality, as well as stability and national continuity. A national language is also instrumental to facilitate development. As one of only few African nations Somalia uses its indegenous language as the official language.

The difficulties to use Somali as the official language were compounded by the fact that there existed no script for the orally transmitted language. So, upon gaining independence, English, Italian, and Arabic were used as official languages. This decision overlooked a rising nationalism among Somalia's population and the use of Somali as an official language became a common national goal. As for the script three alternatives have been discussed. Using a modified Arabic as script for The Somali language has always been a favourite solution for the islamic clerics. Nationalists have preferred the creation of a special script for Somali, the Osmania. The military government under Siad Barre, which came to power in 1969 , finally instituted a script using the Latin alphabet, mostly for pragmatic reasons.

The article chronicles historically the discussion preceeding Barre's decision. Correlations between language problems and emerging national identity become visible, too. 